\title{
ACCESO A LA INFORMACIÓN SOBRE LA PERMANENCIA DEL ESTUDIANTADO UNIVERSITARIO CON MIRAS A LA ACREDITACIÓN \\ ACCESS TO INFORMATION ABOUT THE CONTINUITY OF UNIVERSITY STUDENTS FOR THE ACCREDITATION \\ TRAINING
}

\author{
Volumen 15, Número 1 \\ Enero - Abril \\ pp. 1-30
}

\section{Este número se publicó el $1^{\circ}$ de enero de 2015 \\ DOI: dx.doi.org/10.15517/aie.v15i1.16960}

\author{
Zacarías PereiraVega \\ María Gabriela Regueyra Edelman \\ Julieta Solórzano Salas \\ Viria Ureña Salazar
}

Revista indizada en REDALYC, SCIELO

Revista distribuida en las bases de datos:

CATÁLOGO DE LATINDEX, IRESIE, CLASE, DIALNET, DOAJ, E-REVIST@S, SHERPA/ROMEO, QUALIS, MIAR

Revista registrada en los directorios:

ULRICH'S, REDIE, RINACE, OEI, MAESTROTECA, PREAL, $\underline{\text { CLACSO }}$ 


\title{
ACCESO A LA INFORMACIÓN SOBRE LA PERMANENCIA DEL ESTUDIANTADO UNIVERSITARIO CON MIRAS A LA ACREDITACIÓN ACCESS TO INFORMATION ABOUT THE CONTINUITY OF UNIVERSITY STUDENTS FOR THE ACCREDITATION
}

\author{
Zacarías Pereira Vega ${ }^{1}$ \\ María Gabriela Regueyra Edelman ${ }^{2}$ \\ Julieta Solórzano Salas ${ }^{3}$ \\ Viria Ureña Salazar ${ }^{4}$
}

Resumen: El presente artículo sintetiza la investigación realizada por un equipo interdisciplinario sobre la experiencia de las carreras de Trabajo Social, Farmacia, Orientación y Educación Especial de la Universidad de Costa Rica relacionada con la obtención de información sobre la permanencia de la población estudiantil, esto para atender las compromisos de autoevaluación y la toma de decisiones. También se consideró oportuno indagar sobre el rol de las instancias universitarias que brindan la información requerida. La investigación se realizó en el año 2013, desde una aproximación cualitativa, descriptiva y reflexiva sobre el acceso a la información relacionada con la permanencia del estudiantado. Como resultados se obtienen los requerimientos de información y las principales fuentes, las experiencias positivas y negativas desde la perspectiva de las carreras y de las instancias universitarias, así como un análisis de las implicaciones que tiene para la gestión de las Carreras y la Universidad las limitaciones en el acceso a la información entre las que se destacan los tiempos de respuesta, inconsistencias cuantitativas entre la información aportada por las diversas dependencias los limitados recursos humanos y la carencia de aplicaciones informáticas para dar respuesta a los requerimientos de las carreras. Concluye el artículo insistiendo en que para lograr la calidad de la educación se requiere de un sistema de información que permita dar cuenta de los logros y los retos de las carreras y evidenciar que la población estudiantil ejerce el pleno derecho a la Educación Superior cumpliendo con los compromisos asumidos ante la sociedad costarricense.

Palabras clave: EDUCACIÓN SUPERIOR, ESTUDIANTES, PERMANENCIA, ACREDITACIÓN, UNIVERSIDAD DE COSTA RICA

\begin{abstract}
This article synthesizes the research performed by an interdisciplinary team about the experience of the Social Work, Pharmacy, Counseling, and Special Education careers in the University of Costa Rica, related to the compilation of information about the continuity of the student population. This is done to attend the demands of the self-evaluation and the decision-making process. It was also considered convenient to inquire about the experience of the university offices that provide the required information. The research was carried out in the year 2013, from a qualitative, descriptive and reflexive approach regarding the access to information connected to the continuity of the students. The results are: the need for information and main sources, positive and negative experiences from the perspective of the careers and the university offices, as well as an analysis of the implications the limitations to information access have for the management of the Careers and the University, among which the following stand out: response times, quantitative inconsistencies between the information provided by the dependence, limited human resources, and the lack of technological applications to support the needs of the careers. The article concludes with a reminder that to accomplish the desired quality of education an information system is required, which accounts for the accomplishments and the challenges of the careers, and demonstrates that the student body exercises its rights to Superior Education by accepting their responsibility towards Costa Rican society.
\end{abstract}

Key words: HIGHER EDUCATION, STUDENTS, PERMANENCY, ACCREDITATION, UNIVERSITY OF COSTA RICA

\footnotetext{
${ }^{1}$ Profesor de la Facultad de Farmacia e Investigador del Instituto de Investigación en Educación (INIE), de la Universidad de Costa Rica Dirección electrónica: zacariaspereira@ucr.ac.cr

${ }^{2}$ Profesora de la Escuela de Trabajo Social e Investigadora del Instituto de Investigación en Educación (INIE), de la Universidad de Costa Rica.Dirección electrónica: gabriela.regueyra@ucr.ac.cr

${ }^{3}$ Profesora de la Escuela de Orientación y Educación Especial e Investigadora del Instituto de Investigación en Educación (INIE), de la Universidad de Costa Rica. Dirección electrónica: julieta.solorzano@ucr.ac.cr

${ }^{4}$ Profesora de la Escuela de Orientación y Educación Especial e Investigadora del Instituto de Investigación en Educación (INIE), de la Universidad de Costa Rica. Dirección electrónica viria.urena@ucr.ac.cr
}

Artículo recibido: 21 de mayo, 2014

Enviado a corrección:29 de julio, 2014

Aprobado: 20 de octubre, 2014 


\section{Introducción}

Como parte del proyecto de investigación \#724-B2-006 "Seguimiento a la permanencia de la población estudiantil de la Universidad de Costa Rica", el equipo investigador consideró importante recuperar las situaciones que enfrentaron las carreras de Trabajo Social, Farmacia, Orientación y Educación Especial (participantes en el proyecto), para obtener la información necesaria sobre la permanencia ${ }^{5}$ de la población estudiantil para los procesos de autoevaluación con fines de acreditación, e indagar sobre la experiencia de las instancias universitarias a las que se les solicita la información requerida. En esta línea de trabajo se propuso realizar una investigación que planteó como propósito general: Analizar la experiencia que han tenido algunas carreras e instancias universitarias relacionadas con la obtención de información requerida sobre la permanencia de la población estudiantil para los procesos de autoevaluación con miras a la acreditación en la Universidad de Costa Rica

Los resultados de la investigación se sintetizan de seguido.

\section{Bases teórico - conceptuales}

El tema de la calidad de la educación se ha colocado en los últimos años en un lugar de preferencia en las discusiones sobre la educación superior. Al respecto, en la Declaración de la Conferencia Mundial sobre Educación Superior, se plantea:

La expansión en el acceso de la educación genera desafíos en materia de calidad en la educación superior. Asegurar la calidad es una función vital en la educación superior contemporánea y debe involucrar a todos los actores. La calidad requiere tanto del establecimiento de sistemas de aseguramiento de la calidad y pautas de evaluación, así como la promoción de una cultura de la calidad en el seno de las instituciones.

Deberán implementarse mecanismos regulatorios y de aseguramiento de la calidad que promueva el acceso y creen las condiciones para la culminación de los estudios en todo el sistema de educación superior. (Unesco, 1998, p. 2)

La educación superior, considerada como "un centro de reflexión y formación, de transmisión y construcción de conocimiento, como bien universal, de investigación y extensión sin cortapisas distintas a las de la ética", remite a la concepción de la educación como "derecho fundamental de las personas, como bien universal, como responsabilidad

\footnotetext{
${ }^{5}$ Permanencia de la población estudiantil, se refiere al proceso que sigue el estudiantado desde que ingresa a la Universidad a una carrera específica hasta la graduación
} 
estatal" (Díaz, 2011, p. 141) y su calidad estará orientada por estos principios y no a centrada en la eficiencia como objeto de rentabilidad financiera.

Concebir la educación como un derecho es asegurar

...un camino para la conquista de otros derechos civiles y políticos...la educación consiste, por lo tanto, en el proceso de socialización de la cultura de la vida, en el cual se construyen, se mantienen y se transforman los saberes y los conocimientos. La educación se destina a múltiples sujetos y tiene como objetivo el cambio de los saberes, la socialización y la confrontación con el conocimiento, según distintas aproximaciones, ejercidas por personas de diferentes condiciones físicas, sensoriales, intelectuales y emocionales, que pertenecen a diferentes clases, grupos étnicos, género o procedencia, de la ciudad, de los pueblos o de las aldeas. (Brandao y Leite 2011, citado por Díaz 2011, p. 142)

Las carreras universitarias, mediante la autoevaluación y la acreditación, permiten comprobar el logro de su quehacer en el ámbito social y a su vez transformar sus prácticas mediante los compromisos de mejoramiento. Al considerar la educación como derecho, se debe valorar además del ingreso en igualdad de condiciones, la equidad en la permanencia y graduación. Autores como Martín (2010) plantean que la equidad debe darse en el acceso, la permanencia y el logro de un plan de estudios por parte del estudiantado, es decir, como indica Brovetto, citado por Cardoso (2013), el ejercicio pleno del derecho humano de la educación superior.

En Costa Rica el derecho a la educación superior es un gran reto, ya que persiste la desigualdad y la inequidad en el acceso y la permanencia, como lo plantea el Programa Estado de la Nación en Desarrollo Humano Sostenible (2013, p. 211):

Las oportunidades académicas en el nivel universitario tienen un componente de desigualdad que refleja la concentración de la riqueza que exhibe el país. La tasa de asistencia en los quintiles superiores de la distribución es mucho mayor que la de los grupos de menores ingresos. Además se observan diferencia entre sectores: mientras en las instituciones públicas la relación entre los quintiles quinto y primer es de 2,8 veces, en las privadas es de siete veces. Del total de población de 18 a 24 años que asiste a las universidades estatales un $46,8 \%$ pertenece a los tres primeros quintiles, mientras que en el sector privado la proporción es de 32,4\%. 
La acreditación debería determinar no solo cuánto se acerca una carrera a los parámetros previamente determinados por una agencia acreditadora, sino también cuánto se acerca a los compromisos asumidos ante la Universidad y ante la sociedad, y con ello asumir la autoevaluación y mejora como procesos permanentes, es decir, incorporar la reflexión y la construcción de propuestas para reorientar sus acciones en el cumplimiento de su misión y del derecho a la educación, como garantía de su calidad.

Para la Universidad de Costa Rica (UCR) la calidad académica que se busca es:

...aquella que logra vincular su quehacer con las transformaciones de la sociedad y, por lo tanto, su énfasis se traslada de los productos a los procesos académicos, pues es ahí donde se pueden provocar las medidas correctivas y perfectibles. Lo antes planteado significa, además, una concepción de calidad que se sostiene en el poder democratizador de la educación, y en la posibilidad de enriquecer el desarrollo individual y colectivo mediante la apropiación del saber, la producción de conocimiento, y la contribución al desarrollo social, científico, cultural y tecnológico de los diferentes sectores de la sociedad" (UCR, 2007, p. 29)

Los procesos de autoevaluación y autorregulación de las carreras tienen un impacto positivo en la institución como un todo, ya que al aumentar el número de carreras acreditadas, crece el reconocimiento de la institución y al mismo tiempo, los requerimientos de autoevaluación y los compromisos de mejora generarán cambios graduales en otras instancias universitarias; al respecto, afirma el Programa Estado de la Nación en Desarrollo Humano Sostenible. (2013, p. 236):

La identificación de debilidades con frecuencia remite situaciones más generales del contexto institucional. El análisis efectuado mostró una relación de dos vías en los procesos generados por las actividades tendientes a la acreditación. Por una parte las acciones de autoevaluación y mejora ejecutadas en las carreras o las carreras influyen en la calidad de las instituciones en general y, por otra parte, los cambios organizativos y los planes elaborados en el ámbito institucional tienen un efecto positivo en la calidad de las carreras.

A la Universidad de Costa Rica como institución de educación superior le corresponde, de acuerdo con el artículo 5, incisos c) y e) del Estatuto Orgánico (UCR, 1974, p. 2)

c) Formar profesionales en todos los campos del saber, que integren una cultura 
humanística con su formación especial o profesional [...]

d) Formar personal idóneo que se dedique a la enseñanza, las humanidades, las ciencias, la tecnología, las artes y las letras, para que participe eficazmente en el desarrollo del sistema de educación costarricense.

La Universidad de Costa Rica es una institución de educación superior humanista y democrática, comprometida con el pleno ejercicio de los derechos humanos, como se puede constatar con los artículos mencionados, así como en los principios orientadores establecidos en el Artículo 4 del Estatuto Orgánico, donde se detalla, entre otros, el derecho a la educación superior, la excelencia académica e igualdad de oportunidades, y el respeto a la diversidad de etnias y culturas.

En esta línea humanista, la institución se ha caracterizado históricamente por colocar a la población estudiantil en el centro de su quehacer; desde los años 70, a partir del Tercer Congreso Universitario, se crea la Vicerrectoría de Vida Estudiantil con rango superior en la estructura organizativa, dependiendo directamente de la Rectoría, la cual integra y consolida un conjunto de servicios y programas dirigidos a la población estudiantil, que favorecen la igualdad y la equidad de oportunidades (Universidad de Costa Rica, 2001).

Acercase a las características y necesidades de la población estudiantil y desarrollar acciones que favorezcan el proceso de ingreso, permanencia y graduación, se ha convertido en uno de los retos de las carreras y de los servicios estudiantiles que ofrece la Vicerrectoría de Vida Estudiantil, mediante acciones que favorezcan el desarrollo integral de la población estudiantil y su logro académico (Universidad de Costa Rica, 2001). El conjunto de estas acciones son las que pueden contribuir para que el incremento en la admisión, también impacte el logro terminal. En esta línea, las universidades públicas estatales en el Quinto Convenio de Financiamiento para la Educación, se comprometieron con

....incrementar el acceso, cobertura, permanencias y graduación; mejorar la calidad y relevancia de los programas académicos y los recursos humanos; fortalecer el desarrollo científico y tecnológico, así como la innovación; fortalecer la gestión institucional y la rendición de cuentas (Programa Estado de la Nación en Desarrollo Humano Sostenible, 2013, p. 227)

En el marco de los procesos de autoevaluación -en este artículo siempre se comprende la autoevaluación con miras a la acreditación-, el seguimiento a la permanencia y 
graduación de la población estudiantil es un reto, ya que se requiere un sistema de información que, además de almacenar los datos de la población, permita a las carreras consultar y generar información para la toma de decisiones, como: características demográficas, socioeconómicas, académicas, entre otras que permitan evidenciar el avance, el logro académico y la no continuación de cada estudiante, ${ }^{6}$ de cada cohorte $^{7}$ y de la población en general, lo que solo es posible con un proceso permanente de monitoreo de la información, e implica generar y analizar cada año la información, identificar los retos, realizar acciones de mejora, valorar el impacto de estas acciones, así como plantearse, si es necesario, incorporar nueva información en el sistema, o complementarla con otros esfuerzos, como la consulta a la comunidad universitaria, aspecto que reafirma Erazo (2007, p. 87):

En consecuencia, resulta difícil instaurar sistemas destinados a dar garantías de calidad sin un desarrollo paralelo de capacidades de autoevaluación y autorregulación en las instituciones de educación superior, así como sin el desarrollo de capacidades de generación y análisis de información para la toma de decisiones. De lo contrario, los procesos instalados tienden a burocratizarse, formalizarse y convertirse en procesos de cumplimiento más que en oportunidades de mejora.

Como producto de los procesos de autoevaluación y acreditación, se ha evidenciado desde las carreras la necesidad de contar con información oportuna y sistemática sobre la permanencia de la población estudiantil, que permita dar cuentas del avance y logro académico de cada estudiante que la conforma. Al respecto, se señala como una las principales dificultades encontradas en los procesos de autoevaluación:

...carencia de bases de datos integrales en la Universidad, con la información que requieren las carreras para sustentar dichos procesos. Igualmente, en la mayoría de las unidades académicas, los registros de información son rudimentarios y no están estandarizados (UCR, 2007, p. 38)

La incorporación de la tecnología como herramienta en los procesos administrativos y académicos inicia en la Universidad de Costa Rica con el "Sistema de Información Estudiantiles" (SIE), que administra la Base de Datos Estudiantiles y los procesos del área de

\footnotetext{
${ }^{6}$ No continuación: se refiere a la población estudiantil que no continúa matriculando cursos de la carrera en estudio.

${ }^{7}$ Cohorte: grupo de estudiantes que concretan el ingreso a carrera en un mismo año.
} 
Vida Estudiantil de la Universidad de Costa Rica (UCR, 1998, p. 5). Con el desarrollo y evolución de nuevas plataformas y concepciones de sistemas de información, se plantea desde la Vicerrectoría de Vida Estudiantil y con la colaboración del Centro de Informática, la necesidad de construir un sistema con plataformas abiertas que favorezca la interacción con otros sistemas y bases de datos, la integración y el acceso a la información por parte de los diversos servicios de la Universidad, y ofrecer desarrollos de cara a los múltiples usuarios, como se indica en el documento proyecto: Desarrollo del Sistema Académico Estudiantil (UCR, 1998, p. 9) "Se pretende aprovechar las innovaciones tecnológicas presentes y promover la migración del SIE hacia plataformas informáticas abiertas que nos permitan poner a disposición de los usuarios finales la información de la base de datos estudiantil."

Como una nueva opción, se propone crear el Sistema de Aplicaciones Estudiantiles (SAE), que inició su desarrollo en 1999 y ha sido producto de un esfuerzo progresivo y en etapas. Cada día hay nuevas plataformas, nuevas herramientas y nuevos requerimientos, por lo que continúa creciendo y desarrollándose, utilizando propuestas novedosas para favorecer el servicio a los usuarios, como es el caso de Ematrícula, que constituye una plataforma en línea para que la población estudiantil realice el proceso de matrícula, lo que ha provocado un salto cualitativo en los sistemas de acceso a servicios.

Las bases de datos del SAE guardan información histórica de diversos procesos administrativos generados por oficinas como Registro e Información, Becas y Atención Socioeconómica, Centro de Evaluación Académica, entre otras. La forma de capturar esos datos ha pasado de la digitación manual a la lectora óptica, hasta llegar a la información en línea, en la que el mismo usuario realiza los trámites como matrícula y becas, desde cualquier computadora conectada a la web, independientemente del lugar donde se encuentre.

Sin embargo, a pesar de que la Universidad cuenta con la Base de Datos Estudiantiles, no ha desarrollado aplicaciones para que las carreras tengan acceso a la información necesaria para dar seguimiento a la permanencia y graduación de la población estudiantil. En esta línea, en el Cuarto Informe del Programa Estado de la Educación en Desarrollo Humano Sostenible, se señala como uno los retos que deben superar las universidades "Mejorar sustantivamente la información disponible, generando indicadores de gestión para evaluar el quehacer de las instituciones, alimentar una planificación estratégica de la educación universitaria y fortalecer una vigorosa cultura de rendición de cuentas" (Programa Estado de la Nación en Desarrollo Humano Sostenible, 2013, p. 237) 
Ante este panorama surgen las siguientes interrogantes a las que la investigación se propuso dar respuesta:

- ¿Cuáles son los requerimientos de información sobre la permanencia de la población estudiantil de parte de las carreras?

- ¿Cómo han logrado las carreras obtener información sobre la permanencia de la población estudiantil para los procesos de autoevaluación y toma de decisiones?

- ¿Qué limitaciones encuentran los responsables de las bases de datos estudiantiles para dar respuesta a los requerimientos de información de las carreras?

\section{Proceso metodológico}

\subsection{Tipo de investigación}

El equipo investigador realizó diferentes acercamientos a la comprensión del objeto de investigación propuesto: “¿cómo enfrentan los requerimientos de información sobre la permanencia de la población estudiantil, algunas carreras que realizaron procesos de autoevaluación con fines de acreditación?". Estos requerimientos de información generan una red de interrelaciones a lo interno de la carrera y en el contexto institucional, que conforman un fenómeno complejo, el cual puede impactar de manera positiva o negativa el proceso de autoevaluación; estas premisas coinciden con postulados de la investigación cualitativa, ya que esta

\section{Profundiza en los diferentes aspectos que influyen en determinadas situaciones}

(la negrita es del original). La realidad es holística y polifacética, no es estática, esta se va creando. No existe una única realidad, sino múltiples realidades interrelacionadas; el ser humano es activo en la construcción y determinación de las realidades que encuentra; interesa conocerlas en su dimensión espacial y temporal, el aquí y el ahora es un contexto histórico social determinando. (Cedeño, 2001, p. 7)

En este marco, el equipo investigador reconoce que la realidad y los hechos sociales están conformados por una complejidad de interrelaciones que para su comprensión requieren de una visión global. De ahí que el proceso de aprehensión del objeto de investigación se orientó a la búsqueda de respuestas a las interrogantes formuladas por el equipo investigador, realizando esfuerzos para identificar, comprender e interrelacionar, las concepciones y formas en que los diferentes actores institucionales perciben y enfrentan los requerimientos de información sobre la permanencia de la población estudiantil, para los 
procesos de autoevaluación, en la Universidad de Costa Rica, coincidiendo con el planteamiento de Gurdián (2010, p. 141) “...un hecho científico o cualquier acontecimiento deber ser visto-analizado desde el mundo de relaciones, interacciones de todo tipo que teje a su alrededor. No puede ser simplemente visto desde su fragmentariedad".

Las sesiones de discusión, reflexión y jerarquización iban desde lo particular a lo general, desde la teoría a la realidad, de las expectativas formales a la realidad de los actores, en concreto, desde:

- las carreras en específico y la Universidad como un todo

- Ios lineamientos generales planteados en ambas guías de autoevaluación establecidas por el SINAES, vinculados con la permanencia del grupo de estudiantes de las carreras consultadas, hasta las dimensiones, componentes y evidencias

- Ios datos obtenidos hasta el análisis desarrollado en los informes de autoevaluación relacionados con la permanencia del estudiantado

- los diversos actores que recuperan su experiencia y percepción del objeto

- las evidencias empíricas recuperadas por medio de entrevistas

Estos esfuerzos son característicos del enfoque cualitativo, como lo plantea Cedeño (2001, p. 8)

El punto básico de partida del enfoque cualitativo está centrado en la comprensión de las personas dentro del marco de referencia de ellas mismas, en el contexto de su práctica concreta, de su cotidianidad. Esto sustenta el por qué la comprensión se valora desde una perspectiva holística, totalizadora, no fragmentada, que considera todos los puntos de vista como valiosos y dignos de estudiar; y donde las creencias, juicios de valor y predisposiciones de las y los investigadores deben tratar de apartarse. De esta forma, ningún aspecto de la vida social resulta ser tan frívolo o trivial para no ser estudiado.

El centro de atención de la investigación fue ese conjunto de interrelaciones (fenómenos) generado ante los requerimientos de información sobre la permanencia de la población estudiantil de algunas carreras que realizaron procesos de autoevaluación con fines de acreditación, y no la persona en sí misma, sino, como lo indica Gurdián (2010, p.144):

La investigadora y el investigador cualitativo actúan como facilitadores-técnicos del 
grupo de investigación, y su función adquiere carácter de instrumental, al abolir la separación sujeto/objeto. El grupo es el que asume colectiva y solidariamente la ejecución de la investigación. Las y los sujetos se auto-investigan. Ni ellas ni ellos son el objeto de conocimiento, sino las situaciones, los fenómenos, los acontecimientos, relaciones sociales y la estructura material y simbólica del medio o de la institución.

En síntesis, se realizó un estudio descriptivo - reflexivo bajo el paradigma cualitativo. El proceso investigativo se dio a partir de aproximaciones de aprehensión del objeto de investigación, que no tienen orden secuencial, sino en un ir y venir de la realidad a la reflexión, del análisis textual al conceptual, de las categorías y códigos a la problematización, de las interrelaciones de los actores a la información.

\subsection{Etapas o aproximaciones al objeto de investigación:}

- Definición del problema por investigar, objetivos de investigación y aspectos por abordar

- Discusión teórica: sobre las temáticas vinculadas y definición de posibles categorías de análisis

- Discusión sobre la metodología: selección del tipo de estudio, características y alcances, selección de técnicas de investigación y construcción de instrumentos de acuerdo con los acercamientos al objeto. Se utilizaron técnicas como: entrevista focalizada, comparación de contenidos y análisis documental.

- Recopilación de la información: para esta etapa se elaboraron instrumentos para caracterizar los datos relacionados con la permanencia del estudiantado, que se establecen en los informes de autoevaluación de las carreras consultadas, Escuela de Trabajo Social (2008, 2010), Sección de Educación Especial (2012) y Sección de Orientación (2012). Así como instrumentos para registrar la fecha, número de oficio, tipo de información solicitada y la correspondencia de esta con la evidencia, según el Manual de acreditación y el tipo de información recibida, el cual incorporó fecha, número de oficio, tipo de información solicitada y valoración de la información por parte del equipo investigador.

Para la entrevista focalizada se elaboró una guía con introducción, objetivos, categorías de análisis previas, población participante y el programa de la carrera (acreditada, 
reacreditada y por reacreditar). Cada una de las grabaciones realizadas en las entrevistas semiestructuradas se transcribió. Las personas participantes dieron su consentimiento para realizar la grabación. Dichas actividades se realizaron entre junio y octubre de 2013. Para el registro de las entrevistas se utilizaron códigos para identificar los aportes de cada persona entrevistada, según la instancia que representa, como se detalla en el Cuadro 1:

Cuadro 1. Códigos utilizados en el proceso de investigación, según informante entrevistado

\begin{tabular}{|c|c|}
\hline Códigos & Informante \\
\hline CASE-CS & $\begin{array}{l}\text { Coordinación del Centro de Asesoría Estudiantil de Ciencias Sociales- } \\
\text { CASE Ciencias Sociales }\end{array}$ \\
\hline CASE-S & Coordinación del Centro de Asesoría Estudiantil de Salud- CASE Salud \\
\hline CASED & $\begin{array}{l}\text { Coordinación del Centro de Asesoría Estudiantil a Estudiantes con } \\
\text { Discapacidad CASED }\end{array}$ \\
\hline CEE & $\begin{array}{l}\text { Coordinación de la Comisión de Autoevaluación de la carrera de Educación } \\
\text { Especial }\end{array}$ \\
\hline CF & Decanatura de la Facultad de Farmacia \\
\hline $\mathbf{C O}$ & $\begin{array}{l}\text { Coordinación de la Comisión de Autoevaluación de la Escuela de } \\
\text { Orientación }\end{array}$ \\
\hline CTS & de Trabajo Social \\
\hline OBA & de la Oficina de Becas y $A$ \\
\hline ORI & de la Oficina de Registro \\
\hline
\end{tabular}

Fuente: construcción propia, 2013

- Análisis de la información: se utilizó el software ATLAS-Ti, como herramienta para realizar la sistematización y el análisis textual de la información, para lo cual se subieron como documentos primarios, las transcripciones de las entrevistas y los análisis de documentos. Con esta herramienta se registraron también los comentarios, análisis, conclusiones e interrogantes que surgían en las sesiones de trabajo del equipo investigador, para crear las familias de: documentos primarios, de códigos y de memos.

Las categorías que orientaron la discusión y el análisis de la información se exponen en el Cuadro 2: 
Cuadro 2. Categorías que orientaron el análisis y su respectiva definición

\begin{tabular}{|c|c|}
\hline Categorías & Definición \\
\hline $\begin{array}{l}\text { 1. Requerimientos } \\
\text { de información }\end{array}$ & $\begin{array}{l}\text { Se refiere a la información requerida por la carrera en relación con la } \\
\text { permanencia de la población estudiantil }\end{array}$ \\
\hline Instancias & $\begin{array}{l}\text { Son las oficinas universitarias a las cuales se debe recurrir en busca de } \\
\text { información sobre la permanencia de la población estudiantil al no tener la } \\
\text { carrera la información requerida para el proceso de autoevaluación }\end{array}$ \\
\hline Otras dependencias & $\begin{array}{l}\text { Dependencias externas a la Universidad a las que recurren las carreras } \\
\text { para obtener la información requerida sobre la permanencia del } \\
\text { estudiantado }\end{array}$ \\
\hline Requerimientos & $\begin{array}{l}\text { Necesidades de información sobre el estudiantado de acuerdo con las } \\
\text { guías del SINAES y para la gestión administrativa }\end{array}$ \\
\hline $\begin{array}{l}\text { 2. Metodología } \\
\text { trabajo de } \\
\text { carrera }\end{array}$ & $\begin{array}{l}\text { Estrategias utilizadas por las carreras para obtener información; } \\
\text { incluye técnicas, bases de datos, documentos y fuentes de } \\
\text { información. }\end{array}$ \\
\hline Fuente de información & $\begin{array}{l}\text { Se refiere a las bases de datos, documentos, oficios, informes entre otros } \\
\text { que utiliza la carrera para obtener la información que requiere }\end{array}$ \\
\hline Procedimiento & $\begin{array}{l}\text { Estrategias que utilizó la carrera para obtener la información relacionada } \\
\text { con la permanencia del estudiantado }\end{array}$ \\
\hline 3. Resultados & $\begin{array}{l}\text { Experiencias positivas y negativas durante el proceso de recolección de la } \\
\text { información, así como las implicaciones de los resultados para la carrera }\end{array}$ \\
\hline Experiencias positivas & $\begin{array}{l}\text { Son las respuestas efectivas y oportunas que favorecieron el proceso de } \\
\text { recopilación de información }\end{array}$ \\
\hline Implicaciones-gestión & $\begin{array}{l}\text { Implicaciones para la gestión: las consecuencias que tiene para la gestión } \\
\text { el no contar con la información requerida }\end{array}$ \\
\hline Limitaciones & $\begin{array}{l}\text { Son los obstáculos a los que se enfrentó la carrera para obtener la } \\
\text { información que permitiera responder a los requerimientos del proceso de } \\
\text { autoevaluación con fines de acreditación, con la información de la } \\
\text { permanencia del estudiantado; pueden ser de tiempo, de contenido o } \\
\text { ausencia de información }\end{array}$ \\
\hline Soluciones & $\begin{array}{l}\text { Acciones ejecutadas por la carrera para enfrentar las limitaciones de } \\
\text { información sobre la permanencia del estudiantado }\end{array}$ \\
\hline Limitaciones de la UA & $\begin{array}{l}\text { Se refiere a las dificultades que tienen las unidades académicas en } \\
\text { relación con el procesamiento de la información de su población } \\
\text { estudiantil }\end{array}$ \\
\hline $\begin{array}{l}\text { 4. Instancias } \\
\text { universitarias }\end{array}$ & $\begin{array}{l}\text { Experiencias de las instancias universitarias ante los requerimientos } \\
\text { de las unidades académicas en relación con la información de sus } \\
\text { estudiantes, en el marco de los procesos de autoevaluación }\end{array}$ \\
\hline $\begin{array}{l}\text { Limitaciones de } \\
\text { instancias }\end{array}$ & $\begin{array}{l}\text { Los obstáculos que limitaron brindar de manera oportuna (tiempo) y } \\
\text { pertinente (lo que solicitó) los requerimientos de las carreras }\end{array}$ \\
\hline $\begin{array}{l}\text { Aciertos de las } \\
\text { Instancias }\end{array}$ & $\begin{array}{l}\text { Experiencias positivas de las instancias en relación con la respuestas } \\
\text { dada a la carreras }\end{array}$ \\
\hline $\begin{array}{l}\text { Procedimiento } \\
\text { instancias UCR }\end{array}$ & $\begin{array}{l}\text { Estrategias utilizadas por las instancias para dar respuesta a los } \\
\text { requerimientos de información de las carreras }\end{array}$ \\
\hline
\end{tabular}

Fuente: construcción propia, 2013 


\section{Análisis de los resultados}

A continuación se exponen y discuten los resultados obtenidos, considerando la experiencia de las carreras en los procesos de acreditación y de las instancias que aportan información.

\subsection{Experiencia de las carreras en los procesos de acreditación}

En Costa Rica el Sistema Nacional de Acreditación de la Educación Superior (SINAES) es la institución a la que el Estado costarricense, por medio de la Ley N8256 Sistema Nacional de Acreditación de la Educación Superior, y la Ley № 8798 Fortalecimiento del Sistema Nacional de Acreditación de la Educación Superior, encomendó la misión de dar fe pública de la calidad de aquellas carreras universitarias y parauniversitarias que aprobaron un proceso de autoevaluación, el cual se basa en estándares aceptados tanto a nivel nacional como internacional.

El modelo de acreditación del SINAES, mediante la Guía para la autoevaluación, solicita a las carreras una serie de información referida al ingreso, permanencia y graduación del estudiantado, además de la gestión administrativa que acompaña estos procesos.

Las carreras participantes en el estudio son: Licenciatura en Farmacia, Bachillerato y Licenciatura en Trabajo Social, Bachillerato y Licenciatura en Educación con Énfasis en Orientación y Bachillerato en Educación con Énfasis en Educación Especial. Las 4 carreras se han acreditado con el SINAES; la experiencia de autoevaluación para la acreditación de cada carrera se ha realizado en periodos diferentes.

La carrera de Trabajo Social fue la primera acreditada por dicho organismo en la Universidad de Costa Rica, en 2001 y, en 2006, la reacreaditación. Por su parte, la carrera de Farmacia obtiene la acreditación en 2004 y la reacreditación en 2008. Las carreras Orientación y Educación Especial se acreditan en 2012.

Dicha situación implica que las 2 primeras carreras utilizaron el Manual de Acreditación Oficial de Carreras de Grado del SINAES de 2000, y las otras 2 emplearon el de 2009. En ambos manuales se encuentran temáticas vinculadas con la permanencia de la población estudiantil, como se aprecia en el Cuadro 3: 
Cuadro 3: Requerimientos de información en los Manuales de Acreditación Oficial de Carreras de Grado del Sistema Nacional de Acreditación de la Educación Superior, de 2000 y 2009

\section{Manual de Acreditación Oficial de Carreras de Grado del Sistema Nacional de Acreditación de la Educación Superior de 2000}

- Estadísticas sobre estudiantes que solicitan ingreso, matriculados, traslados de carrera y admisión de estudiantes de otras instituciones - La carrera o programa debe disponer, de manera fidedigna y eficiente, de información respecto a los estudiantes (características sociodemográficas, índices de deserción, índices de graduación, índices de permanencia, rendimiento académico por cursos y profesor carga académica, utilización de servicios, entre otros) y utilizar esta información en la toma de decisiones para la ejecución y desarrollo del plan de estudios.

Fuente: Elaboración propia, 2013
Manual de Acreditación Oficial de Carreras de Grado del Sistema Nacional de de Acreditación de la Educación Superior de 2009

- Serie histórica de las matrículas y de los graduados de los últimos cinco años

- Lista de estudiantes procedentes de otras universidades a quienes se les reconocieron o equipararon cursos, según universidad, carrera de procedencia y cursos reconocidos

- Distribución de los estudiantes de la carrera a quienes se les han equiparado créditos, según porcentaje de créditos reconocidos con respecto al total de créditos del plan de estudios

- Distribución de los estudiantes admitidos en los últimos cuatro años según sexo, nacionalidad, edad, condición de discapacidad si la presentan e institución educativa de procedencia

- Porcentaje de estudiantes de la carrera becados, con ayudas económicas 0 financiamiento, que opinan que estos apoyos recibidos son suficientes

- Disponibilidad de estadísticas sobre el uso de los servicios académicos y estudiantiles

- Estadísticas anuales disponibles sobre el estudiantado y sus características

- Distribución de los estudiantes matriculados según promedio anual obtenido en los últimos cuatro años por nivel

- Distribución de los estudiantes graduados en los últimos cuatro años según la modalidad de trabajo final de graduación presentado

- Existencia de una base de datos con información de los graduados

Es evidente que el manual de 2009 solicita a las carreras una mayor cantidad de evidencias que permiten una mejor comprensión de las características presentadas por el estudiantado durante permanencia y su graduación.

Al consultar a las personas entrevistadas de cada una de las carreras, sobre los requerimientos de información de la población estudiantil que demanda este modelo, hacen referencia a aspectos tales como: cantidad de hombres y mujeres matriculados por año, cantidad de estudiantes que ingresan por año, promedio ponderado del estudiantado, uso de los servicios de la universidad, cantidad de cursos matriculados, títulos reconocidos, cantidad 
de traslados de carrera y de estudiantes graduados en los últimos 4 años. La persona entrevistada en Trabajo Social agrega ...instrucción de cómo se aseguró la carrera el cumplimiento de la estadística de bienestar, la calidad de la carrera y cómo apelan los estudiantes...(CTS)

Por su parte, la persona entrevistada en el CASED comenta que las carreras solicitan información de la población con discapacidad que ha ingresado a la carrera en los últimos 3 años, el tipo de discapacidad y el colegio de procedencia, además de información general sobre la accesibilidad en el proceso de admisión y aspectos relacionados con acceso a la educación universitaria.

Las personas entrevistadas por cada carrera indican que también solicitan información sobre el uso de los servicios por parte del estudiantado de la carrera, de ahí que demanden dicha información al Sistema de Bibliotecas documentación e información (SIBDI), CASE, OBAS, ORI, entre otros, como se ilustra en la siguiente cita ... porque no había cambiado la guía, [el] requisito de graduación, [las] estadísticas [sobre estudiantes] que solicitaron ingreso, matriculado, trasladado de carrera, admisión, eso sí lo da la oficina de servicios, asesoría y facilidades que se le brinda al estudiante en salud... (CTS)

Para atender las necesidades de información de la población estudiantil, las carreras organizan el listado de requerimientos previamente, según mencionan las personas entrevistadas: ...fuimos armando el esqueleto y diciendo qué necesitamos,... (CF) y...la comisión de la Escuela, hizo un inventario de todas las evidencias que ocupábamos... (CEE)

Como primer paso, se identifica la información que se requiere y se establecen las posibles fuentes y estrategias para recolectarla. En cuanto a las fuentes de información, las carreras utilizan 3 tipos:

- Fuentes internas, que se refieren a la información que se encuentra en las carreras. Entre esas, la oficina o persona encargada de asuntos estudiantiles, que custodia

- la información de la población estudiantil de la carrera:...la información que se incluye proviene de una fuente interna, el registro es posible obtenerlo, ya que se cuenta con las nóminas de personas graduadas... (CEE)

También, como fuentes internas, se recurre a las diferentes comisiones existentes en las carreras, como la Comisión de Credenciales: ...la información que aparece en el documento de autoevaluación se redacta a partir de la información facilitada por la Comisión de Credenciales, ... (CO) 
- Por su parte, las fuentes externas a la carrera se refieren a las que son parte del contexto universitario.

En estas fuentes externas se mencionan las instancias universitarias como la ORI, la OBAS, el Centro de Evaluación Académica (CEA), el SIBDI, la Oficina de Orientación, específicamente el CASE de Ciencias Sociales, el de Salud y el CASED. La carrera de Trabajo Social consultó al Instituto de Investigaciones en Ciencias Económicas.

- Fuentes externas a la Universidad son aquellas fuera del contexto de la Universidad de Costa Rica; a modo de ejemplo, la carrera de Farmacia solicitó datos al Consejo Nacional de Rectores (CONARE) y al Colegio de Farmacéuticos de Costa Rica, para responder a la caracterización de la población graduada.

La estrategia utilizada por las carreras para recolectar información de las fuentes internas y las externas, es el envío de oficios. Por su parte, la instancia consultada utiliza como medios de respuesta, los formatos electrónico o impreso.

Para conocer la información del estudiantado y su opinión sobre los servicios que ofrece la Universidad, como los de becas, los complementarios y los de la Oficina de Orientación, entre otros, las carreras utilizaron la técnica del cuestionario.

Asimismo, las carreras realizan otros esfuerzos adicionales, como... generar información a pie, lo que les genera inversión de tiempo y recursos... (CF).

Las personas entrevistadas en los procesos de recolección de datos sobre la población estudiantil, se enfrentaron experiencias positivas y negativas. Entre las experiencias positivas consideradas como las respuestas efectivas y oportunas que favorecieron el proceso de recopilación de información de las carreras, se mencionaron las siguientes:

- Las respuestas oportunas del SIBDI y la OBAS, ya que fueron instancias que entregaron la información solicitada una semana después u 8 días calendario, de acuerdo con los oficios enviados y recibidos; cumpliendo además con los contenidos solicitados, como se aprecia en las siguientes afirmaciones:

...por ejemplo [el] SIBDI nos mandó un CD con todos los datos [de las referencias bibliográficas] específicas para Educación Especial, qué era lo que había, cómo se interrelacionaba [en] un documento toda la posición del SIBDI [con la carrera] (...) hasta uno queda súper seguro de esa información... (CEE) 
- El esfuerzo realizado por el personal de la carrera de sistematizar la información que tenían, así, por ejemplo...entonces nosotros empezamos a sacar de nuestras listas, de las actas que saca Laurita la secretaria, empezamos nosotros a inventariar todo a mano... (CEE)

Por otra parte, entre las experiencias negativas que enfrentan las carreras para cumplir con los requerimientos de información, se señalaron las siguientes:

- A pesar de que se cuenta con cierta información, como se señala en la siguiente cita... nos piden ingreso a carrera, cupos y esas cosas si nos las dan... (CTS); no se tuvo acceso a la información directamente del SAE que se solicita en la guía del SINAES, ya que no se cuenta con las aplicaciones específicas para dar respuesta a los requerimientos. Por tal razón, cada carrera debe solicitar los datos a las dependencias que los generan:

...el otro día en una reunión (...) que convocó el Consejo Universitario - es parte de las quejas que las Unidades decimos- que estamos en estos procesos de acreditación porque hay información, pero hay que pedirla a la oficina de registro para que nos la puedan facilitar, porque no hay acceso de las unidades académicas a la información que pide la guía del modelo de SINAES... (CF)

... pero esa información es de la ORI, uno puede tener acceso a lo que vos conoces que el SAE nos puede dar acceso, pero no esa información tan específica, sexo, edad, lugar de procedencia... (CO)

- El periodo de respuesta por parte de algunas instancias fue prolongado, principalmente la Oficina de Registro e Información, la cual requirió hasta 3 meses para responder las solicitudes de la carrera, según lo indican las personas entrevistadas y la revisión realizada a los oficios de solicitud y respuesta. Al respecto, las personas entrevistadas manifiestan:

...tardaron demasiado en darnos la respuesta, fue algo muy lento, fue impreciso...los oficios se iban y pasaban 2 semanas, un mes sin respuesta, algunos hasta más, un oficio fueron 3 meses que nadie sabía nada del oficio....tal vez Registro nos iba a mandar la información, ellos ahí a su plazo, pero lo que pasa es que también, es la precisa, y todo el conjunto, porque entonces SINAES tenía que con 6 meses ya no nos iba a recibir la información y por otro lado, imagínate que íbamos a tener que esperar 6 meses para que Registro nos diera la información y todo aprieta...(CEE) 
- Diferencias entre los datos enviados por las instancias y los que tiene la carrera, por las metodologías utilizadas y los periodos de análisis, entre otros, tal y como se detalla...no había precisión en la información, porque Registro nos daba unos datos y nosotros manejábamos otros datos... (CO)

- Los datos recibidos de las instancias son estadísticas generales que no permiten identificar a cada estudiante, o complementar la información con la que ya se tiene, de manera que se le pueda dar seguimiento para favorecer su permanencia y graduación; al respecto se menciona:

...la Oficina de Registro no está en capacidad de dar la información de manera pertinente y oportuna, entonces a uno le mandan estadísticas y qué tipo de estadística y como igual no tienen históricos, entonces no sabe uno de qué estudiantes están hablando, si es el matriculado... (CO)

Los aspectos señalados como experiencias negativas tienen consecuencias para la gestión de la carrera y la Universidad, principalmente porque:

- No se logra una visión integral de la población estudiantil de la carrera:...pero no podíamos decir, de qué colegio son los estudiantes, qué se yo, cuántos años tienen, no podíamos decir nada de ellos, porque en realidad no los conocíamos, nos dimos cuenta que no conocíamos a nuestros estudiantes... (CEE)

- No se cuenta con datos oportunos para la toma de decisiones y dar respuesta a las interrogantes que surgen sobre la población estudiantil; al respecto, las personas entrevistadas indican:

...porque sería información importante que yo tengo que conocer para ver bueno qué pasa,...yo empecé a ver quiénes no hicieron y por ejemplo hay estudiantes que están en dos carreras, entonces dejaron esta, por ejemplo desde el 2011 no matriculan nada y están en la otra... (CF)

- Las carreras en particular y la Universidad en general, no están preparadas para generar la información que requiere el proceso de autoevaluación, como se describe: ...la Universidad de pronto no estaba preparada, para las demandas que iban a tener las carreras que estaban en procesos de autoevaluación, con miras a la acreditación... (CO) 
- Las carreras no tienen claro a cuál instancia solicitar la información, como por ejemplo, la que se ilustra a continuación, relacionada con la accesibilidad que ofrecen las carreras a la población con alguna discapacidad:

...me mandan a preguntar a mí si el edificio de Ciencias Sociales es accesible... ustedes tienen los usuarios ahí, ustedes saben que el baño no es accesible entonces ustedes tienen esa información, ustedes me la mandan a pedir a mí, y yo no voy a ir a investigar si es accesible, o sea yo tengo la noción y puedo llevar un chequeo de lo que es accesible o no en esta universidad... (CASED)

Ante las dificultades señaladas para obtener la información requerida, las carreras incorporaron nuevos procedimientos que fueron incluidos en los compromisos de mejora, y a partir de los cuales se debe hacer rendición de cuentas anualmente, como se mencionó en una de las entrevistas:

...y es una debilidad que tenemos que ver cómo subsanar, porque para este informe tenemos que tener la base de datos y ahora el compromiso es que la base de datos se mantenga actualizada... (CF)

Las carreras también han desarrollado instrumentos para sistematizar la información relacionada con la permanencia y graduación del grupo de estudiantes. Como parte de las acciones de mejora, han creado sus propias bases de datos, duplicando con esto los esfuerzos institucionales, como se ejemplifica en las siguientes afirmaciones:

...este medio año que terminan los muchachos su internado...lo que hicimos es que haya una formulita que cuando ellos llegan a entregar papeles aquí, para que se les dé la carta indicando que ya concluyeron, que ya solo queda pendiente el acto de graduación, entonces el compromiso es que aquí en Asuntos Estudiantiles, pidan la información para que quede actualizada, digamos la información de ellos de correo electrónico... (CF)

Como parte del proceso de investigación, se revisaron los informes de autoevaluación de las carreras participantes, identificando la información que solicita el SINAES en las guías correspondientes, y se encontró que de manera general, se presenta información que se relaciona con las evidencias solicitadas, pero no con la precisión que se indica, como por ejemplo: el SINAES solicita la nota promedio anual obtenida, pero en el informe de 
autoevaluación aparece la nota promedio de matrícula obtenida, o bien, se solicita la nota promedio anual obtenida por nivel en los últimos 4 años, y la carrera reporta la nota promedio global de los estudiantes de la carrera, y no la diferencia por nivel.

\subsection{Experiencias de las instancias que aportan información}

Con el objetivo de obtener una visión integral sobre las implicaciones que tienen para la Universidad los requerimientos de información sobre la población estudiantil, específicamente para los procesos de autoevaluación, el equipo investigador incorporó la experiencia de las diferentes instancias universitarias.

En respuesta a la solicitud de las carreras, las instancias como el SIBDI, el CASE de Salud y la OBAS, envían información estadística generada a partir de los procesos que realizan como parte de su plan de trabajo diario. Además, a esta información se adjuntan documentos sobre los tipos de servicios que estas oficinas brindan a la comunidad estudiantil universitaria, como se evidencia a continuación:

entonces se le hace un listado a la decana, con todos los proyectos y servicios que se le están ofreciendo a la población estudiantil, la información solicitada es una información muy general, no nos piden información puntual con nombre de estudiantes, cantidad de estudiantes, ni nada de eso.... (CASE SALUD)

Por su parte, la OBAS facilita la información en forma de estadísticas globales, la cual no identifica a los estudiantes individualmente y es remitida por correo electrónico a las carreras que la solicitan.

... nos lo piden por nota, de hecho nos llega una carta y de inmediato yo paso la instrucción para que vayan al área de becas socioeconómicas que ahí está el área de estudios de estadísticas, y ya ahí piden las estadísticas, se remiten e incluso por correo electrónico para montar la respuesta y entonces se le hace llegar la respuesta a la unidad, no es un procedimiento que implique operativamente una asignación de recursos exhaustiva por lo menos hasta este momento, lo importante es que, como es vía sistema, tenemos dos personas estadísticas en la oficina, eso no nos da problema y la generación se hace... a este nivel; total de estudiantes becados y por género que es lo que nos puede dar el sistema ... (OBAS)

En el caso de la información estudiantil generada y custodiada por la ORI, el 
procedimiento debe iniciar con un oficio enviado por la carrera al CEA, instancia encargada de informar a la ORI acerca de dichos requerimientos. Al parecer, este procedimiento surge por el gran esfuerzo y recursos que debe destinar la ORI para satisfacer las necesidades de información de las carreras.

EI CASED indica que revisan otras fuentes de información estudiantil en procura de cumplir con la solicitud.

...lo que ya te digo, siempre tenemos que sacar a pie lo del colegio, porque ya te digo, en esa base mía de permanencia no lo tengo porque lo puedo chequear en SAE como vos decís... busqué el expediente en vivo y a todo color, lo metemos... (CASED)

En relación con las experiencias de las instancias para facilitar la información de las carreras, se pueden agrupar en aciertos y limitaciones. Los aciertos corresponden a las experiencias positivas que identifican las instancias en relación con las respuestas dadas a las carreras:

- La disponibilidad de información, pues instancias universitarias como el CASE-SALUD y la OBAS, refieren estar preparadas para facilitar la información solicitada por las carreras, como se expone:

... en ese sentido para nosotros no es un problema poderles brindar esa información, porque tenemos todo organizado, hay un plan de trabajo que organiza todo nuestro quehacer.... (CASE SALUD)

- La disposición para modificar procedimientos y dar respuesta oportuna de acuerdo con los requerimientos de las carreras, instancias como el CASED y los CASE de las distintas áreas, han subsanado situaciones mediante la coordinación conjunta de acciones, al respecto:

... porque hace como 2 o 3 años... hicimos un esfuerzo de unificar lo que íbamos a informar, porque todo el mundo te preguntaba lo mismo y entonces un CASE explicaba lo mismo con palabras de una forma y el otro diferente.... (CASED)

De la misma manera, la ORI refiere que sus informáticos ganan experiencia cada vez que se les solicita información y han logrado actualizar los datos de las personas graduadas. A continuación se lee una cita que confirma lo señalado... hoy en día ese requerimiento del control de graduados y seguimiento de graduados que hacen para acreditación para esta oficina ya es una fortaleza, porque nos ha obligado a actualizar esa información.... (ORI) 
Como se indicó, las instancias enfrentan también limitaciones para ofrecer de manera oportuna y pertinente, los requerimientos de las carreras; hicieron mención a:

- Insuficiente recurso humano, pues las instancias universitarias cuentan con una cantidad de personal limitado y con funciones muy variadas.

... pero yo sí te puedo hablar de lo cualitativo, lo que tengo son 2 profesionales en informática, más la jefe de desarrollo informático, esos 2 profesionales $B$... lo otro es que tengo un recurso informático que también es encargado en redes y una web máster, ese es el equipo con que trabajamos, ahora ellos dan soporte a todos los procesos diarios que tiene la institución, usted sabe que esta oficina está cargada de soportes informáticos, estamos a la vanguardia en aplicaciones Web, ellos nos dan ese soporte, uno de nuestros compañeros profesional $B$ que tenemos, le da soporte también de colaboración al Instituto de Investigaciones Psicológicas a lo largo y ancho del proceso de admisión, ..... (ORI)

Lo anterior lleva a la ORI a priorizar las respuestas a los oficios sobre solicitudes de información estudiantil, sin que ello demerite o afecte la calidad de los servicios que brinda a la comunidad universitaria.

... es que hay que darle prioridad a las solicitudes, si los reportes que hoy en día saca la oficina y que son de acceso a las unidades académicas,... requerimientos del SINAES por ejemplo, o el órgano de acreditación para las ingenierías que es un órgano canadiense, tenemos que sacar a nuestro recurso y destinarlo a otro tipo de labores sin que se demerite la calidad de servicios de los estudiantes de las Unidades académicas o [el que] las autoridades demandan día a día... (ORI)

- Calidad de la información, en tanto hay dificultad por parte de la ORI para ofrecer datos de la información estudiantil antes de 2007. A partir de ese año hubo un cambio a otro sistema informático, y pueden ofrecer ese tipo de información, según lo explica el representante de la ORI

... sin embargo hay algunas otras limitaciones, yo le puedo hablar de calidad de datos en información del año 2007 a la fecha, cuándo nos pasamos a Java, cuando ustedes nos hacen un reporte, por ejemplo del trabajo que ustedes están realizando del año 2002, vos me has escuchado que yo no puedo garantizar la calidad de los datos y es mi responsabilidad también en ese sentido, hubo migraciones, cambios de sistemas, algunas de las variables que hoy en día existen no fueron solicitadas en ese momento, 
no existía la doble carrera tan fuerte como hoy existe, tenemos más de 6 mil estudiantes en doble carrera hoy en día, estudiantes que están en dos o más recintos, o en un recinto en dos carreras diferentes, no hay una normativa clara sobre la doble carrera, entonces tenemos que aplicar la normativa institucional sobre una variable que no estaba contemplada ... (ORI)

Los procesos de acreditación de las carreras requieren una estrecha coordinación con las oficinas que ofrecen servicios y generan información, en la que hay un intercambio de información, de manera que las oficinas se constituyan en un apoyo para la carrera. Se menciona en las entrevistas, experiencias en las cuales las oficinas administrativas que ofrecen servicios son convocadas a reunión con pares externos, sin embargo, estas no conocen el documento final de autoevaluación. A pesar de que el informe contiene un apartado en el que se incluye la opinión de la población estudiantil sobre los servicios que se ofrecen, se han dado situaciones en las cuales los pares externos consultan a los representantes de las oficinas administrativas su visión sobre lo que opinó la población estudiantil de la carrera, y las personas de dichas oficinas desconocen lo manifestado por esta población; comenta una de las personas entrevistadas sobre una sesión con pares externos:

...las señoras se fueron así [refiriéndose a una de las personas que fungía como par externo], no le estoy preguntando que hacen, así decía la señora con un amor, no le estoy preguntando qué hacen, me parece que es excelente, estoy preguntando: ¿cómo van a resolver lo que los estudiantes dicen? Y nosotros decíamos: ¿ Y qué dicen los estudiantes? Ajá y por ejemplo ¿qué dicen los estudiantes? Decíamos nosotros... llegó un momento que ella dice yo creo o estoy tratando de entender que ustedes no saben qué les estoy preguntando yo ... (CASED)

De la misma manera, en las sesiones que realizan las carreras y las instancias con los pares externos, es fundamental que ambas instancias universitarias manejen los resultados del proceso de autoevaluación relacionados con la población estudiantil, sus expectativas y satisfacción en cuanto a los servicios que se ofrecen, integrando la información como una unidad, y no de forma fragmentada, como al parecer se maneja actualmente.

Algunas instancias universitarias manifiestan su inconformidad o extrañeza cuando las carreras, en sus procesos de autoevaluación, les solicitan información estudiantil que no es la que normalmente se solicita: 
... pero cuando yo ya paso a la base de permanencia a no ser que yo lo requiera me voy al SAE y busco la información, pero no es un dato que yo diga, tengo ingresados tantos y tantos del colegio, ya eso no, yo lo hago en admisión porque tengo que ubicar dónde les hago entrevistas, pero una vez ingresados esos datos, entonces nos ha pasado: ¿qué es eso?, vea lo que nos piden, jefa, ¿qué hacemos? ... (CASED)

Existe información estudiantil muy sensible que es custodiada por las instancias, , y se debe manejar con cuidado, respetándose la privacidad de cada estudiante. Este derecho es garantizado por las leyes de la República y respaldado plenamente por la Universidad de Costa Rica, por cuanto: ...yo me estremezco cuando un director me llama y me dice que necesitamos información, cómo explicarlo que desde la privacidad del estudiante no se puede. (OBAS)

... la Oficina Jurídica ha mantenido un criterio que la información que se custodia, que tiene que ver en materia de calificaciones, de títulos, de dirección, de diplomas, de correos electrónicos, números telefónicos; está protegido y esto es un artículo de la Constitución Política... (ORI).

Por su parte, el CASE-Salud reconoce la importancia de que la información estudiantil que ellos generan y custodian, pueda segmentarse para las carreras, pues ... permiten ver la necesidad de ir afinando más, estas estadísticas que nosotros podemos dar, para poderlas discriminar mejor, no en totales, sino tantos eran de Farmacia, tanto de Micro, tantos de Medicina .... (CASE Salud)

La experiencia de autoevaluación y acreditación no deja de representar cierto nivel de cuestionamiento -e incertidumbre- sobre la manera de hacer el trabajo en todos los niveles, no solo en la carrera, sino también en las mismas instancias universitarias que realizan una labor muy importante y que, a su vez, generan y custodian información sensible del grupo de estudiantes, la cual exige una coordinación estrecha entre las dependencias universitarias, para evitar situaciones como la que se refleja en la siguiente cita:

... le voy a decir algo, ahí arriba estábamos y le puedo asegurar que todos hicimos un gran esfuerzo por no tener que decir abiertamente, que no conocíamos los datos, pero tuvimos que hacerlo, porque era tan incisiva la pregunta, tal cosa tal cosa, entonces yo le decía a la gente, yo creo que para efectos de esto y de otros ustedes tienen que retomar, nosotros llegamos aquí como a ciegas, pensamos que vinimos a contar [lo] que hacemos, pero cuando nos sentamos vinimos a responder cosas muy concretas 
que dijeron los estudiantes de nuestros servicios y no sabemos qué es, entonces usted llega a la reunión y va desarmado o va a decir lo que sea, ... (CASED)

Por su parte, la OBAS pretende implementar visitas a las distintas carreras, y en concreto al profesorado de estas, a efectos de socializar la información estudiantil y que se conozca, mediante estadísticas, el perfil socioeconómico de sus estudiantes. Así se pretende sensibilizar al personal docente, con el propósito de impactar en la oferta académica, la confección de horarios y las consecuencias negativas de una matrícula ordinaria incompleta.

... que identifique con el perfil del estudiante, e incluso yo quiero incorporar dentro de los planes de trabajo el poder hacer visitas a las unidades académicas y ojalá dentro de lo que es la asamblea de profesores que se tenga compartir estadísticas para que ellos conozcan el perfil de estudiantes con que ellos tienen que identificarse porque de ahí va hacer muy importante también que ellos se sensibilicen desde como confeccionan horarios, la oferta académica, que conozcan el impacto que tiene para el estudiante becado una oferta académica en matrícula ordinaria incompleta y que la vayan a completar con inclusión que eso tiene un efecto muy negativo para el estudiante en términos de la entrega de los beneficios ... (OBAS)

Por otro lado, es un tanto cuestionable que la información del estudiantado no se le facilite a la autoridad académica de la carrera, pues estando la carrera en un proceso de autoevaluación, es inconcebible pensar que no pueda conocer integralmente a sus estudiantes: ¿de dónde vienen?, ¿quiénes son?, ¿cuáles son sus condiciones? Todo para efectos de dar seguimiento, así como proponer soluciones oportunas y reales ante eventuales situaciones durante su permanencia en la Universidad para la toma de decisiones, que es potestad última de la autoridad académica de la carrera.

... requiero conocer cuál es la lista de estudiantes con beca once ... se le dice que se le puede indicar un número de estudiantes, pero no se puede mandar una lista con los nombres y carné de los estudiantes por el derecho a privacidad del estudiante porque ya en material legal rige esta condición que solamente con autorización del estudiante o para fines judiciales, sin embargo, hubo un pronunciamiento de la Sala Cuarta recientemente, donde se dice que sí se puede otorgar información del estudiante siempre y cuando él no sea identificado .... (OBAS) 
Lo anterior evidencia la necesidad de que las carreras cuenten con sus propios sistemas de información relacionada con los datos de estudiantes y población graduada, debido a que los que tiene disponibles la Universidad, resultan limitados.

\section{Conclusiones y recomendaciones}

A la luz de las interrogantes que orientaron la presente investigación, se concluye que: Los requerimientos de información sobre la permanencia de la población estudiantil de las carreras en el proceso de autoevaluación para la acreditación, surgen de la información que se solicita en los manuales que entrega la instancia acreditadora, en este caso el SINAES. De la revisión de las guías del SINAES, se identifica que la información solicitada es: número de estudiantes matriculados, características (sexo, edad, nacionalidad, institución educativa de procedencia, condición de discapacidad), rendimiento académico (promedios obtenidos) y número de graduados, toda de los últimos 4 años previos a la autoevaluación, así como estadísticas, descripción y opinión sobre los servicios académicos estudiantiles que utiliza la población. La información solicitada caracteriza la población estudiantil de los últimos 4 años, pero no permite dar cuentas sobre el avance, el logro académico y la no continuación de cada cohorte y de la población en general, ni la relación entre los servicios de apoyo utilizados por la población estudiantil, y el logro académico.

Con el propósito de satisfacer los requerimientos de información sobre la permanencia de la población estudiantil, para los procesos de acreditación, las carreras se organizan identificando la información requerida y las posibles fuentes internas y externas, siendo el oficio la estrategia más utilizada para solicitar la información. Entre las fuentes internas a la carrera, destacan las Comisiones de Credenciales, de Trabajos Finales de Graduación y la Oficina de Asuntos Estudiantiles. Entre las fuentes externas se indican instancias universitarias y extrauniversitarias. Como parte de las instancias universitarias se encuentran, principalmente, la Oficina de Registro e Información, la Oficina de Becas y Atención Socioeconómica, la Oficina de Orientación y el Sistema de Bibliotecas, Documentación e Información. Como instancias externas a la Universidad, a las cuales se les solicitó información, se menciona un colegio profesional y el CONARE. Se evidencia, con la presente investigación, que en el proceso de recolección de datos por parte de las carreras, estas parecieran no tener claro en algunos casos, a cuál instancia solicitar lo que necesitan. 
Al identificar aciertos y limitaciones relacionados con la obtención de la información requerida sobre la permanencia de la población estudiantil, para los procesos de acreditación, se establecen como aciertos las respuestas efectivas y oportunas, como las del SIBDI y la OBAS. Lo anterior, por cuanto favorecieron el proceso de recolección de la información, ya que se consiguió en tiempo razonable y acorde a las demandas.

Por otra parte, entre las limitaciones que enfrentaron las carreras para recolectar la información, se indica que el tiempo de respuesta de algunas instancias fue mayor a lo esperado, lo que evidencia que no están preparadas para generar los datos requeridos en los procesos de autoevaluación. Se evidenció también con la investigación, que generar información desde diferentes instancias de manera separada, provoca inconsistencias cuantitativas entre la información aportada por las diversas dependencias.

En relación con la experiencia de las instancias universitarias, entre los logros se indica que han realizado esfuerzos para dar respuesta a la información solicitada por las carreras, así como que han tenido la disponibilidad para modificar los procedimientos y brindar una respuesta oportuna a lo requerido por las carreras. Sobre esto mismo, se mencionan como limitaciones: insuficiente recurso humano y carencia de aplicaciones informáticas para dar respuesta a los requerimientos de las carreras.

También es importante indicar que aunque el SINAES solicita estadísticas generales sobre el estudiantado, esto no permite tener una visión integral de esa población en los procesos de ingreso, permanencia y graduación, que sirva como base para la toma de decisiones por parte de las carreras, o para la puesta en marcha de acciones que garanticen el éxito académico de esta. Es por ello que los requerimientos de información estudiantil por parte de las carreras trascienden los establecidos en las guías del SINAES, como se evidencia en los compromisos asumidos en el Plan de Mejoramiento de la Escuela de Trabajo Social (2006): "Investigar los comportamientos de la deserción estudiantil y los tiempos de duración de la carrera", en los documentos Compromiso de Mejoramiento, Sección de Educación Especial (2012) "Creación de un Sistema de información del estudiantado de la carrera de Bachillerato en Ciencias de la Educación con énfasis en Educación Especial" y Sección de Orientación (2012) "Creación de un sistema de información anual relacionada con el estudiantado".

El equipo investigador recomienda que para satisfacer las necesidades de información sobre la permanencia de la población estudiantil, que permitan evidenciar el avance, el logro académico y la no continuación de cada estudiante, de cada cohorte y de la población en 
general para los procesos de autoevaluación con fines de acreditación, y para la toma de decisiones de las carreras, se requiere que:

- Las carreras tengan acceso a los datos específicos de la población estudiantil para la toma de decisiones en la gestión curricular y administrativa, mediante un sistema de información que permita almacenar y sistematizar la información requerida por la carrera.

- Las demandas de información estudiantil oportuna y sistemática, por parte de las carreras, deben tomarse con toda la seriedad del caso, para efectos de que coadyuve integralmente en la toma de decisiones, lo cual se justifica ampliamente en una responsable y adecuada gestión universitaria.

- El tema de ingreso, permanencia y graduación estudiantil, desde una perspectiva del derecho a la educación, implique el trabajo conjunto de todos los actores universitarios, y una gestión críticamente informada y responsable, que facilite las condiciones objetivas para el desarrollo integral del grupo de estudiantes.

- Las carreras realimenten a las oficinas administrativas sobre los resultados obtenidos en los procesos de autoevaluación y autorregulación, relacionados con su quehacer.

Finalmente, se insiste en que para lograr la calidad de la educación, se requiere de un sistema de información que permita dar cuenta de los logros y los retos de las carreras, demostrado que la población estudiantil ejerce el pleno derecho a la educación superior, lo que trasciende la información que solicita el SINAES, al cumplir con la igualdad en el ingreso y la equidad en la permanencia y graduación de la población estudiantil, garantizando así los compromisos asumidos por la carrera y la Universidad ante la sociedad costarricense.

\section{Referencias}

Cardozo, Gisela. (2013). La educación superior universitaria ¿un derecho humano? $X$ Jornadas de Sociología. Facultad de Ciencias Sociales, Universidad de Buenos Aires, Buenos Aires. Recuperado de http://www.aacademica.com/000-038/129

Cedeño, María. (2001). Aportes de la investigación cualitativa y sus alcances en el ámbito educativo. Revista Electrónica Actualidades Investigativas en Educación, 1(1), 1-23. Recuperado http://revista.inie.ucr.ac.cr/ediciones/controlador/Article/accion/show/articulo/aportesde-la-investigacion-cualitativa-y-sus-alcances-en-el-ambito-educativo.html 
Díaz, Luis. (2011). La educación superior: entre derecho y mercancía. Criterio Jurídico Garantista, 3(5), 140-153. Recuperado de http://www.fuac.edu.co/recursos web/documentos/derecho/revista criterio/articulosgar antista5/8Luis Diaz.pdf

Erazo, Iris. (2007). Gestión de calidad en la educación superior. En Gilberto Alfaro, Patricia Ramírez-Fischer y Matthias Wesseler (eds.), Promover la universidad: cinco estrategias y un dilema, la gestión de la calidad y cambio en la Educación superior: experiencias de Unicambio XXI (pp. 79-88). San José, C.R.: UNED.

Escuela de Trabajo Social. (2006). Plan de Mejoramiento de la Carrera de Bachillerato y Licenciatura en Trabajo Social de la Universidad de Costa Rica 2006-2014. San José, Costa Rica: Escuela de Trabajo Social, Universidad de Costa Rica.

Escuela de Trabajo Social. (2008). Informe de reacreditación de la Carrera de Bachillerato Licenciatura en Trabajo Social de la Universidad de Costa Rica. San José, Costa Rica: Escuela de Trabajo Social, Universidad de Costa Rica.

Escuela de Trabajo Social. (2010). Informe de reacreditación de la Carrera de Bachillerato y Licenciatura en Trabajo Social de la Universidad de Costa Rica. San José, Costa Rica: Escuela de Trabajo Social, Universidad de Costa Rica.

Gurdián, Alicia. (2010). El Paradigma Cualitativo en la Investigación Socio-educativa. San José: Editorial de la Universidad de Costa Rica.

Martin, Michaela. (2010). Equity and quality assurance: can they come together? An introduccion to the problematic. En Michaela Martin (ed.), Equity and quality assurance a marriage of two minds (pp. 23-37). Paris: International institute for educational planning.

Programa Estado de la Nación en Desarrollo Humano Sostenible. (2013). Cuarto Informe Estado de la Educación. San José, Programa Estado de la Nación.

Sección de Educación Especial. (2012). Compromiso de Mejoramiento de la Carrera de Bachillerato en Ciencias de la Educación con énfasis en Educación Especial de la Universidad de Costa Rica 2012-2016. San José, Costa Rica: Universidad de Costa Rica.

Sección de Educación Especial. (2012). Informe de Autoevaluación de la Carrera de Bachillerato en Ciencias de la Educación con énfasis en Educación Especial de la Universidad de Costa Rica 2012. San José, Costa Rica: Universidad de Costa Rica.

Sección de Orientación. (2012). Compromiso de Mejoramiento de la Carrera de Bachillerato y Licenciatura en Ciencias de la Educación con énfasis en Orientación de la Universidad de Costa Rica 2012-2016. San José, Costa Rica: Universidad de Costa Rica.

Sección de Orientación. (2012). Informe de Autoevaluación de la Carrera de Bachillerato y Licenciatura en Ciencias de la Educación con énfasis en Orientación de la Universidad de Costa Rica 2012. San José, Costa Rica: Universidad de Costa Rica. 
Sistema Nacional de Acreditación de la Educación Superior. (2000). Manual de acreditación, convocatoria del año 2000. San José, C. R: SINAES.

Sistema Nacional de Acreditación de la Educación Superior. (2009). Manual de Acreditación Oficial de Carreras de Grado del Sistema Nacional de Acreditación de la Educación Superior. San José, C. R: SINAES

UNESCO. (1998). Declaración Mundial sobre la Educación Superior en el siglo XXI: visión y acción.

Recuperado

de http://www.unesco.org/education/educprog/wche/declaration spa.htm

Universidad de Costa Rica. (UCR). (1974). Estatuto Orgánico de la Universidad de Costa Rica. San José, Costa Rica: Consejo Universitario, Universidad de Costa Rica.

Universidad de Costa Rica. (UCR). (1998). Desarrollo del Sistema Académico Estudiantil. Centro de Informática (Publicación interna). San José: Universidad de Costa Rica.

Universidad de Costa Rica (UCR). (2001). Reglamento General de la Vicerrectoría de Vida Estudiantil. San José, Costa Rica: Consejo Universitario, Universidad de Costa Rica.

Universidad de Costa Rica (UCR). (2007). Lineamientos conceptuales y procedimientos generales sobre Autoevaluación-Autorregulación en la Universidad de Costa Rica. Centro de Evaluación Académica (Publicación interna). San José: Universidad de Costa Rica. 